\title{
Spontaneous Rupture of the Left External Iliac Vein: Case Report
}

\author{
Xue-Rong Chen, Wei-Zhong Zhang ${ }^{*}$, Dong-Yi Xin \\ Department of Surgery, Taizhou First People’s Hospital, Taizhou, China \\ Email: "zhangweizhong11@hotmail.com
}

Received May $7^{\text {th }}, 2013$; revised June $9^{\text {th }}, 2013$; accepted June $16^{\text {th }}, 2013$

Copyright (C) 2013 Xue-Rong Chen et al. This is an open access article distributed under the Creative Commons Attribution License, which permits unrestricted use, distribution, and reproduction in any medium, provided the original work is properly cited.

\begin{abstract}
Spontaneous rupture of the iliac vein is rare clinical emergency. Sudden onset, hypertension, and abdominal distention with a nonpulsatile mass in the iliac fossa are the chief symptoms. We reported another case of spontaneous rupture of the left external iliac vein diagnosed intra-operatively and successfully treated with direct suturing. And related literatures were reviewed. Possible etiology and optimal treatment were discussed.
\end{abstract}

Keywords: Spontaneous; Rupture; Iliac Vein; Surgery; Thrombosis

\section{Introduction}

The rupture of an iliac vein is rare, life-threatening, and it mostly results from trauma or injury during pelvic surgery. Spontaneous rupture of the iliac vein is even more unusual, and most cases were diagnosed only intraoperatively and surgery was often associated with substantial mortality and morbidity [1-20]. Only Cho et al. reported successful conservative treatment of spontaneous rupture of the left common iliac vein [14]. We reported a case of spontaneous rupture of the left external iliac vein diagnosed intra-operatively and successfully treated with surgery. And related literatures were reviewed and discussed.

\section{Case Report}

A previously healthy 51-year-old female peasant with no recent history of trauma was referred to our emergency room, because she presented with sudden coma after 4-day's spasm of left calf. Her husband told us on their arrival that the patient showed coma 30 minutes ago after she complained of sudden onset of left lower abdominal pain, and at the same time he found there was progressive distention of lower abdomen. On admission, her blood pressure could not be detected and her pulse rate was 122 beats per minute, breath rate 21 beats per minute. On physical examination, she looked pale, conjunctiva was pale, and there was a solid mass in the left lower abdomen and diffuse swelling of the left lower extremity.

\footnotetext{
*Corresponding author.
}

Laboratory tests revealed a haemoglobin level of $68 \mathrm{~g}$ per liter and a haematocrit level of 25.1\%. Other blood chemistry parameters were within the normal range.

After intensively supportive care, she was recovered consciously, blood pressure reached $70 / 45 \mathrm{~mm} / \mathrm{Hg}$, but the solid mass became much larger and tender. Bed-side Ultrasound scan revealed a heterogeneous mass in the left lower pelvic cavity, which was not related to uterine and ovary. An abdomino-pelvic CT (GE, US) scan without injection of contrast medium revealed a huge retroperitoneal haematoma in the left lower abdomen and pelvic cavity. On the basis of CT findings, spontaneous retroperitoneal haematoma was suspected (Figures 1 and 2).

Emergency laparotomy was performed by lower midline transperitoneal approach. A huge retroperitoneal haematoma measuring $12 \times 10 \times 6.5 \mathrm{~cm}$ was evacuated. The haematoma contained large amounts of clotted venous blood. The left common iliac vein was exposed. A tear of approximately $1.5 \mathrm{~cm}$ was noted at the posteriormedial wall of the left common iliac vein, but there was no active bleeding at the site of rupture because thrombus was found to occlude the lumen of the left common iliac vein completely. The laceration was closed using continuous 5-0 Prolene sutures.

Anticoagulation with low molecular weight heparin was started shortly after surgery. But the patient showed continuous swelling of left lower extremity and Color Doppler ultrasound revealed deep vein thrombosis. The postoperative course was uneventful and the patient was discharged 11 days after surgery with oral administration 


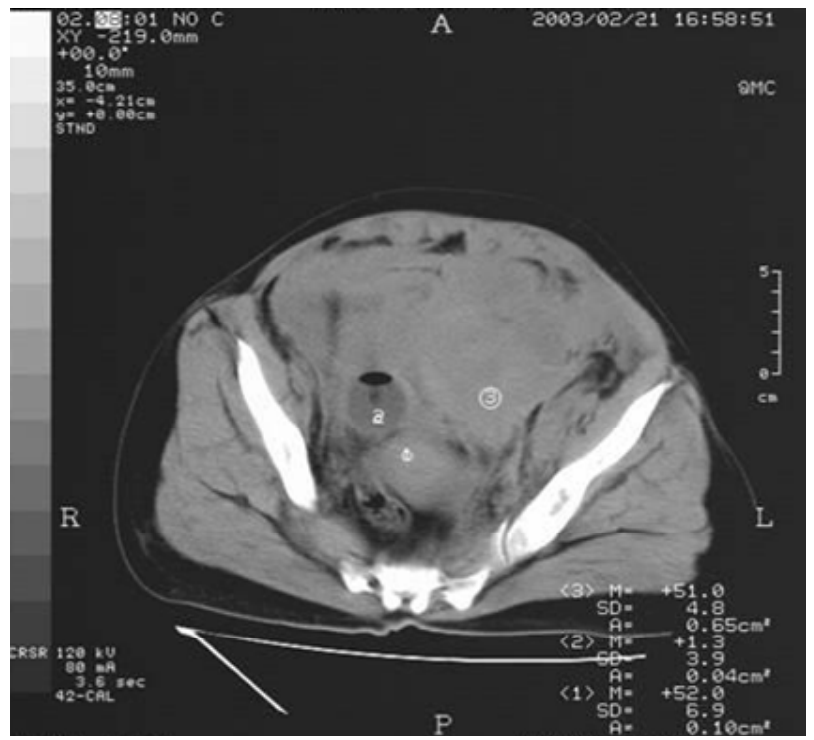

Figure 1. Pelvic CT scan shows a heterogenous mass in left iliac fossa.

of Warfarin for a year. Follow-up Doppler ultrasound showed the patent deep vein of left extremity and she returned to normal work 4 months after discharge.

One year after the initial operation the patient came back to our hospital with mild femoral venous regurgitation on Doppler ultrasound finding and without apparent swelling of the affected lower extremity. And before the submission of the manuscript we interviewed her on telephone. She was very satisfied with the outcome and said that she had been living a happy life during the past decade.

\section{Discussion}

Rupture of iliac vein usually comes from pelvic operations like rectum cancer dissection or injuries due to traffic accidents. In our case, the patient did not present any obvious causes of injury or other possible underlining accident that could contribute to the laceration of iliac vein wall. Therefore, we classified it as spontaneous rupture of iliac vein.

Several hypotheses have been proposed for the possible causes of spontaneous rupture of the iliac vein. The predominant occurrence in middle-aged or elderly women reflects that the estrogen might play a role. With decrease of estrogen level, elasticity of veins will be decreased, which contributes to the laceration of veins. Secondly, a sudden rise in intra-luminal pressure in a segment of the vein between the inguinal ligament and

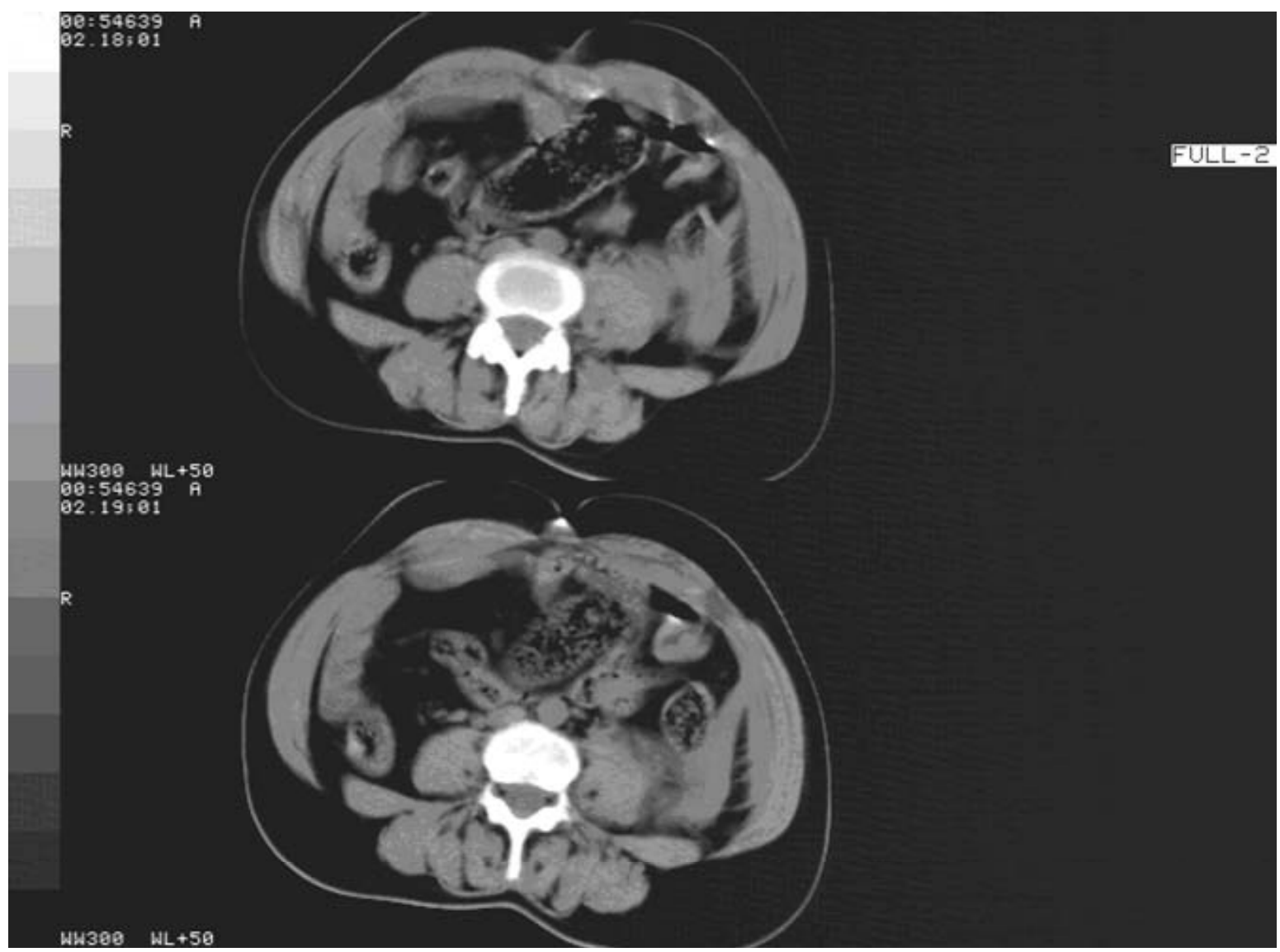

Figure 2. Pelvo-abdominal CT scan shows normal aorta with edema of fascia surrounding psoas major muscle. 
the right common iliac artery could be a contributing factor, which could explain the phenomenon that the rupture occurs predominantly on the left side. Thirdly, thrombophlebitis could result in weakening of the venous wall and might be involved in venous rupture $[12,14]$.

Majority are middle-aged or elderly women, predominately on the left side. Sudden onset of hypotension, lower abdominal pain and distension with a non-pulsatile mass in the iliac fossa, and oedema of the lower extremity of the affected side, without a history of recent trauma, are the clinical pictures found in most cases of spontaneous rupture of the iliac vein $[11,19]$. Because hypovolaemic shock is the chief clinical sign, emergency exploratory surgery is required in most cases of rupture of the iliac vein. However, most cases in the literatures were operated under the misdiagnosis of a ruptured aortic aneurysm or emergency of unknown causes [11,20]. CT might play an important role in this regard. In our case, the pre-operatively CT scan was intended to exclude the diagnosis of abdominal aorta aneurysm.

For the treatment, all of the cases except that reported by Cho et al. [14] underwent operative treatment. Of the reported cases, five patients died after surgery: two after vein ligation and three after vein repair [11,12]. The choice of treatment consists of supportive care, immediate stop of bleeding and maintaining venous patency. Depass et al. [8] also emphasized that prompt resuscitation and emergency surgical management were essential in the treatment of spontaneous rupture of the iliac vein, because, in most cases, hemodynamic stability could not be achieved after resuscitation, and ischemia of the affected limb occurred in some patients. Primary repair with postoperative anticoagulation appears to decrease the risk of post-operative deep vein thrombosis. According to literatures, repair was achieved by direct suture in most cases, with insertion of stent in only two cases [16, 19]. However, post-operative thromboembolic complications were frequently encountered in many cases, which demonstrated a high incidence of thrombosis after repair.

Conservative treatment might be a therapeutic alternative to surgery in selected cases with no evidence of further bleeding. However, according to a report by Cho et al. [14], deep vein thrombosis developed 3 days after conservative treatment. We presume that the risk of developing thrombotic complications may be reduced only when normal venous flow by stent placement was accomplished, as shown in the two cases $[16,19]$.

Spontaneous rupture of the iliac vein is a vascular emergency that should be managed promptly. High clinical alert and emergency exploration with direct repair in middle-aged or elderly women presenting with left-sided retroperitoneal haematoma, deep vein thrombosis, or hypovolemic shock might improve the prognosis, and intravenous stent placement with post-operative anticoagu- lation could diminish the thrombotic complications.

\section{REFERENCES}

[1] S. Hill, P. J. Billings, R. T. Walker and J. A. Dormandy, "True Spontaneous Rupture of the Common Iliac Vein," Journal of the Royal Society of Medicine, Vol. 83, No. 2, 1990, p. 117.

[2] H. Van Damme, G. Hartstein and R. Limet, "Spontaneous Rupture of the Iliac Vein,” Journal of Vascular Surgery, Vol. 17, No. 4, 1993, pp. 757-758. doi:10.1016/0741-5214(93)90121-2

[3] S. Nishida, K. Arikawa, M. Yamashita, et al., "Spontaneous Rupture of Left External Iliac Vein: A Case Report,” Nihon Geka Gakkai Zasshi, Vol. 94, No. 4, 1993, pp. 424-426.

[4] S. M. Majeed and P. J. Pheils, "Spontaneous Rupture of the Left External Iliac Vein,” The British Journal of Clinical Practice, Vol. 47, No. 6, 1993, pp. 109-110.

[5] M. Yamada, M. Nonaka, N. Murai, H. Hanada, M. Aiba, M. Funami, et al., "Spontaneous Rupture of the Iliac Vein: Report of a Case,” Surgery Today, Vol. 25, No. 5, 1995, pp. 465-467. doi:10.1007/BF00311830

[6] G. Plate and P. Qvarfordt, "Idiopathic Rupture of the Iliac Vein. Case Report,” European Journal of Surgery, Vol. 161, 1995, pp. 611-612.

[7] B. C. Lin, R. J. Chen, J. F. Fang, K. E. Lin and Y. C. Wong, "Spontaneous Rupture of Left External Iliac Vein: Case Report and Review of the Literature," Journal of Vascular Surgery, Vol. 24, No. 2, 1996, pp. 284-287. doi:10.1016/S0741-5214(96)70106-4

[8] I. E. DePass, "Spontaneous Common Iliac Vein Rupture: A Case Report,” Canadian Journal of Surgery, Vol. 41, No. 6, 1998, pp. 473-475.

[9] D. Gad, M. V. Thomsen and H. G. Johannsen, "Spontaneous Rupture of the Left External Iliac Vein. A Complication of Deep Vein Thrombophlebitis?” Ugeskrift for Leger, Vol. 161, No. 48, 1999, p. 6639.

[10] N. Gaschignard, Y. Le Paul, T. Maouni and P. D. Le Priol, "Spontaneous Rupture of the Left Common Iliac Vein," Annals of Vascular Surgery, Vol. 14, No. 5, 2000, pp. 517-518. doi:10.1007/s100169910096

[11] D. Pedley, J. Nagy and N. Nichol, "Spontaneous Iliac Vein Rupture: Case Report and Literature Review,” Journal of the Royal Colleges of Surgeons of Edinburgh, Vol. 47, No. 2, 2002, pp. 510-511.

[12] S. Jazayeri, E. Tatou, N. Cheynel, F. Becker, R. Brenot and M. David, "A Spontaneous Rupture of the External Iliac Vein Revealed as a Phlegmasia Cerulea Dolens with Acute Lower Limb Ischemia: Case Report and Review of the Literature,” Journal of Vascular Surgery, Vol. 35, No. 5, 2002, pp. 999-1002. doi:10.1067/mva.2002.121569

[13] N Kismoune, JC Eicher, S Jazayeri, F André, B Terriat, M. David, et al., “'Spontaneous' Rupture of the Left Iliac Vein Complicating Cockett's Syndrome,” Archives des Maladies du Coeur et des Vaisseaux, Vol. 96, No. 4, 2003, pp. 347-350.

[14] Y. P. Cho, Y. H. Kim, J. Ahn, S. Choi, H. J. Jang, S. G. 
Lee, et al., "Successful Conservative Management for Spontaneous Rupture of Left Common Iliac Vein,” European Journal of Vascular and Endovascular Surgery, Vol. 26, No. 1, 2003, pp. 107-109. doi:10.1053/ejvs.2002.1940

[15] T. W. Kwon, S. M. Yang, D. K. Kim and G. E. Kim, "Spontaneous Rupture of the Left External Iliac Vein," Yonsei Medical Journal, Vol. 45, No. 1, 2004, pp. 174176.

[16] S. R. Zieber, B. R. Mustert, M. F. Knox and B. C. Fedeson, "Endovascular Repair of Spontaneous or Traumatic Iliac Vein Rupture,” Journal of Vascular and Interventional Radiology, Vol. 15, No. 8, 2004, pp. 853-856. doi:10.1097/01.RVI.0000128811.75493.CC

[17] Y. Yasuga, N. Hayashi, Y. Nagai and Y. Sasako, "Spontaneous Rupture of the Iliac Vein," Canadian Journal of Surgery, Vol. 47, No. 2, 2004, p. 471.
[18] H. Tannous, F. Nasrallah and M. Marjani, "Spontaneous Iliac Vein Rupture: Case Report and Comprehensive Review of the Literature,” Annals of Vascular Surgery, Vol. 20, No. 2, 2006, pp. 258-262. doi:10.1007/s10016-006-9003-5

[19] Y. H. Kim, S. M. Ko and H. T. Kim, "Spontaneous Rupture of the Left Common Iliac Vein Associated with MayThurner Syndrome: Successful Management with Surgery and Placement of an Endovascular Stent,” British Journal of Radiology, Vol. 80, No. 956, 2007, pp. e176-e179. doi:10.1259/bjr/69371933

[20] I. H. Kim, G. R. Chon, Y. S. Jo, S. B. Park and S. D. Han, "Spontaneous Left External Iliac Vein Rupture," Journal of the Korean Surgical Society, Vol. 81, No. Suppl 1, 2011, pp. S82-S84. doi:10.4174/jkss.2011.81.Suppl1.S82 